\title{
Principle of Self-adaptive Emergence of Wave-particle Duality, Solution to Crisis of a Particle's Simultaneously Passing Double Slits and Objective Criteria Distinguishing Classical and Quantum Particles
}

\author{
C. Huang ${ }^{1,2, *}$, Yong-Chang Huang ${ }^{3,4,+}$ and Yi-You Nie ${ }^{4,++}$ \\ 1. Lawrence Berkeley National Laboratory, 1 Cyclotron Road, Berkeley CA 94720, USA \\ 2. Department of Physics and Astronomy, Purdue University, 525 Northwestern Avenue, W. \\ Lafayette, IN 47907-2036, USA \\ 3. Institute of Theoretical Physics, Beijing University of Technology, Beijing, 100124, China \\ 4. Institute of Theoretical Physics, Jiangxi Normal University, Nanchang, 330022, China
}

\begin{abstract}
This paper uncovers that quantum uncertain principle makes the single particle with global property have no certain path, and then wave of quantum particle can simultaneously do pass the double slits. The two subwaves after passing Young's double slits are entanglement, they may form interference of subwaves. Consequently, we find a kind of quantum probabilistic entanglements with Wheeler's delayed choice. Quantum particles such as photons, electrons, neutrons, protons etc mean that wave of the quantum particle can simultaneously do pass through Young's double slits, rather than individual quantum particle may pass through Young's double slits at the same time. When considering wave property, we cannot consider particle property (Just as in the photoelectric effect, considering the particle nature of the system, people cannot consider wave property, otherwise the photoelectric effect cannot appear). Therefore, this paper discovers that the ability of single photon to hit electrons out in photoelectric effect is complementarily equivalent to the ability of wave of the single photon to simultaneously pass through Young's double slits in wave-particle duality. Objective criteria for distinguishing classical and quantum particles are discovered and objectively give the applicable realm of quantum mechanics for the first time. The crisis of the single particle's simultaneously passing through Young's double slits, which has been plaguing physicists in the whole world up to now for decades, is solved, in which the studies are classified as classical and quantum particles, the classical particle and quantum particle wave cannot and can pass the Young's slits, respectively. This paper discovers both the new physics mechanism of passing the double slits of the wave with the amplitude of 4-dimensional momentum representation wave function reflecting particle nature and the principle of self-adaptive emergence of wave-particle duality, and then using the principle, this paper gives both direct explanations to the current experiments and new predictions of new some experiments for wave-particle duality. All the deduced results here are consistent with all relevant physics experiments.
\end{abstract}

Key words: quantum mechanics, wave-particle duality, Young's Double Slits, interference of double subwaves, quantum probabilistic entanglement

\section{I.Introduction}

In modern physics, the double slit experiments show that microscopic particles can display both classical particle and wave properties, and they show the probabilistic nature of phenomena *Email: c.huang0@hotmail.com; + email: ychuang@bjut.edu.cn; ++email: nieyiyou@jxnu.edu.cn 
of quantum mechanics [1].

In 1801, the double slit experiment was first finished with light by T. Young [2], after a long time, electrons display the same behavior in 1927 [2].

A coherent light source, e.g., a laser beam, lights up one plate pierced by two parallel slits, behind the plate, the light of passing through the slits is observed on a screen [2,3]. The experiments demonstrate that particle doesn't form the interference pattern when one detects which slit the particle passes through, the particle forms the interference pattern when one doesn't detect which slit the particle passes through. These results show the principle of wave-particle duality $[4,5]$.

Feynman said that all of quantum mechanics can be gleaned from carefully thinking through the implications of this single experiment [6], and he presented that if detectors were placed before each slit, the interference pattern would disappear [6].

Ref.[7,8] used single electron and biprism to show that each electron interferes with itself as predicted by quantum theory in 1974. Readers of Physics World voted the single-electron version of the experiment as the most beautiful experiment [9].

The double-slit experiment with electrons and real slits is eventually performed by the original scheme from Feynman, they emit single electrons onto the double slits, and via collecting the transmitted electrons with a single-electron detector, the double-slit interference pattern is shown [10].

For antimatter, Ref.[11] demonstrated single particle interference. Photons, electrons, atoms and even some molecules are measured as a single pulse, the waves of these particles describe the probabilities of absorbing the particles at a specific place on the screen [12-14].

The which-way experiment displays the complementarity principle that photons can show as either particles or waves, but cannot be observed as both at the same time [15]. In the history of quantum mechanics, despite the importance of the thought experiment, until the 1970s, technically feasible realizations of the thought experiment were presented [16]. In order to illustrate various aspects of complementarity, multiple experiments have been performed [17].

In 1987, a performed experiment $[18,19]$ produced the results that the information could be gotten regarding a particle's going which path.

Wave-particle duality of C60 molecules was investigated [20], and Plasmon-Assisted Two-Slit Transmission was explored in Young's Experiment [21].

Wheeler's delayed choice experiments showed that after a particle passes through the slits, extracting "which path" information at the slits can seem to retroactively alter its previous behavior [22].

The new quantum universe is illustrated in Ref.[23]. The discovery that particles are discrete packets of energy with wave-like properties resulted in quantum mechanics dealing with atomic and subatomic systems.

Although quantum theory has achieved the great successes in many aspects, there still is the hard crisis of the single particle's simultaneously passing through Young's double slits, the hard crisis has been plaguing us up to now for decades, this paper wants to give the solution to the crisis.

For example: according to current quantum mechanics, any material can have wave-particle duality, therefore, the physical phenomenon in fig. 1 can exist. But in real world, we don't see such a physical phenomenon, which is an interesting example of the fundamental crisis in the theory of 
quantum mechanics. This paper wants to solve this kind of crisis.

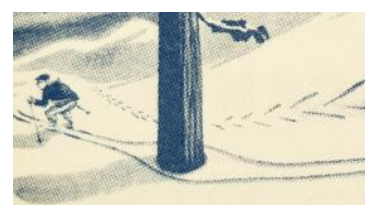

Fig. 1 From Charles Addams, The New Yorker, 1940

The arrangements of this paper are: Sect. two gives studies on the crisis of single particle's simultaneously passing through Young's Double Slits; Sect. three investigates plane wave's simultaneously passing through both Young's Double Slits and any many slits; Sect. four shows quantum uncertain principle's making the single particle with global property have no certain path, and then makes the single particle wave simultaneously do pass the double slits., Sect. five gives a general theory of interference pattern appearance from a kind of quantum probabilistic entanglement; Sect. six shows discussions, summary and conclusions.

\section{Studies on the crisis of single particle's simultaneously passing through Young's double slits}

In photoelectric effect, light waves cannot knock electrons out; and in single photon's passing through young's double slit experiment, single photon cannot pass through Young's double slits at the same time, namely, the two physical processes, respectively, reflect one aspect of wave-particle duality of quantum particle. On the other hand, in photoelectric effect, photons can knock electrons out; in Young's double slit experiment, single photon light wave can pass through Young's double slits at the same time, then the two physical processes are complementarily equivalent in wave-particle duality of quantum particle. That is, in wave-particle duality of quantum particle, the first and the second cases use the particle property and the wave property respectively. Namely, photon can show as either particle or wave, but cannot be observed as both at the same time for a physics process.

We now generally show them by mathematical exact deduction.

In 4-dimensional momentum representation of quantum mechanics, when considering wave function $\phi(\vec{p}, E)$ of momentum representation, one has [24]

$$
\psi(\vec{r}, t)=\frac{1}{(2 \pi \hbar)^{2}} \int_{-\infty}^{\infty} \phi(\vec{p}, E) e^{i(\vec{p} \cdot \vec{r}-t E) / \hbar} d \vec{p} d E=\frac{1}{(2 \pi \hbar)^{3 / 2}} \int_{-\infty}^{\infty} \varphi(\vec{p}, t) e^{i \vec{p} \cdot \vec{r} / \hbar} d \vec{p}
$$

Eq.(2.1) is Fourier transformation of $\phi(\vec{p}, E)$ about the plane wave energy $\mathrm{E}$ and momentum $\vec{p}$, from the four-dimensional momentum representation state vector $\phi(\vec{p}, E)$ to the projection of the plane wave basic vector $e^{i(\vec{p} \cdot \vec{r}-t E) / \hbar}$ and making integration for getting $\psi(\vec{r}, t)$, which make $\psi(\vec{r}, t)$ have not only the characteristics of the probabilistic state vector of the particle but also the characteristics of the plane wave, i.e., make $\psi(\vec{r}, t)$ have the state 
vector characteristics of wave-particle duality. Because the momentum representation state vector $\phi(\vec{p}, E)$ is nonlocal, it also reflects that the system has the global characteristics of momentum $\vec{p}$ and energy $E$, this global property can be the integrity of the particle.

Using Eq.(2.1), we have wave function of momentum representation at time $t$

$$
\varphi(\vec{p}, t)=\frac{1}{(2 \pi \hbar)^{1 / 2}} \int_{-\infty}^{\infty} \phi(\vec{p}, E) e^{-i t E / \hbar} d E
$$

Further using Eq.(2.1), we have two subwave functions through Yaung's double slits

$$
\psi\left(\vec{r}_{j}, t\right)=\frac{1}{(2 \pi \hbar)^{2}} \int_{-\infty}^{\infty} \phi(\vec{p}, E) e^{i\left(\vec{p} \cdot \vec{r}_{j}-t E\right) / \hbar} d \vec{p} d E=\frac{1}{(2 \pi \hbar)^{3 / 2}} \int_{-\infty}^{\infty} \varphi(\vec{p}, t) e^{i \vec{p} \cdot \vec{r}_{j} / \hbar} d \vec{p} .
$$

where $j=1,2$ show the up slit and down slit, respectively, in Young's Double Slits in Fig. 2.

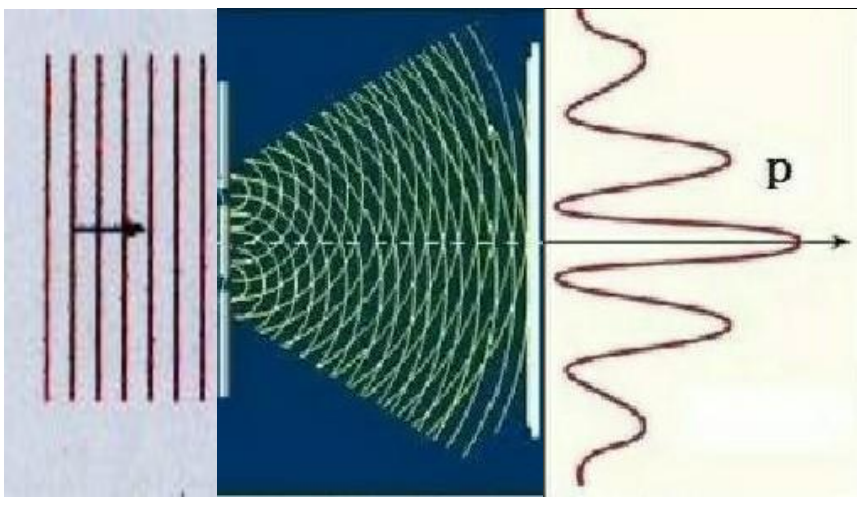

Fig. 2 Interference of single particle plane wave in Young's double slit experiment

The superposition density function of the two subwaves, e.g., see ref.[22], in fig. 2 is just eq.(5.2), the interference terms of the two subwaves in Fig. 2 are just the third term and fourth term in eq.(5.2).

On the other hand, using Huygens' Principle, one has the basic wave analysis:

Every point of a wave front may be considered the source of secondary wavelets that spread out in all directions with a speed equal to the speed of propagation of the waves. What this means is that when one has a wave, he can view the "edge" of the wave as actually creating a series of circular waves. These waves combine together in most cases to just continue the propagation, but in some cases there are significant observable effects. The wave front can be viewed as the line tangent to all of these circular waves [25] .

These properties are exactly conforming to the plane wave properties of the single particle, thus single particle plane wave can simultaneously pass through Young's Double Slits in fig. 2, Eq.(2.3) just generally give the both subwave functions that simultaneously pass through two slits $s_{1}$ and $s_{2}$ in Young's Double Slits, respectively, the two subwave functions have the same amplitude $\phi(\vec{p}, E)$ for some certain $\vec{p}, E, e^{i\left(\vec{p} \cdot \vec{r}_{j}-t E\right) / \hbar}(j=1,2)$ are just two plane subwave functions in Eq.(2.3), and the two probabilistic wave functions in Eq.(2.3) integrate for ( $\vec{p}, E)$ 
from negative infinite to positive infinite, i.e., having considered all possibility, which make the two expressions (2.3) exact.

The global property of particle does not allow the single particle to pass through Young's double slits at the same time, in reality, the interference of individual particle wave is observed, which just show single particle wave simultaneously does pass the double slits, but all theories up to now cannot solve the hard crisis of single particle's simultaneously passing the double slits.

Since the wave function $\phi(\vec{p}, E)$ of the momentum space reflecting the global properties of the particle obtained in this paper is to be integrated from minus infinity to positive infinity, momentum space is nonlocal. During this period, the plane wave can pass through the two slits, because their probability wave functions (2.3) are projections of the particle's four dimensional momentum state vector function $\phi(\vec{p}, E)$ onto the plane waves $e^{i\left(\vec{p} \cdot \vec{r}_{j}-t E\right) / \hbar}$, and the integral from minus infinity to positive infinity occurs simultaneously. So, physics law allows them to happen at the same time, thus we see the interference of a single particle wave through the double slits.

Plane wave of the single particle simultaneously passes through Young's double slits and causes interference, its particle property makes it have no definite path by uncertain relation from wave nature of the particle, it reflects the characteristics of the wave. These specific novel physics details are reflected out by concrete computations in following two sections.

\section{Plane wave's simultaneously passing through both Young's Double Slits and any many slits}

In the double-slit experiment, a beam of particles shot through two slits into the detection screen can produce light and dark diffraction patterns, they show that these particles interfere with each other like classical waves. If only one particle passes through the double slits at a time, it is not interference between particles, but each particle interferes with itself. If we want to detect which slit the particle passes through, the interference pattern disappears. We can either get the interference pattern or detect which slit the particle passes through, we cannot obtain the both at the same time.

Because the two subwave functions have the same amplitude $\phi(\vec{p}, E)$ for some certain $\vec{p}, E$, i.e., $\vec{p}, E$ are certainty, the positions are uncertainty $[24,26]$. We concretely calculate as follows:

Due to

$$
\Delta \hat{p}^{2}=\overline{(\hat{p}-\overline{\hat{p}})^{2}}=\overline{\hat{p}^{2}}-\overline{\hat{p}}^{2},
$$

when considering plane wave $\psi_{p}=c e^{i(x p-E t) / \hbar}$, one has

$$
\int_{-L}^{L} \psi_{p}^{\dagger} \psi_{p} d x=\int_{-L}^{L} c^{\dagger} c d x=|c|^{2} 2 L=1 \rightarrow c=\frac{e^{i \alpha}}{(2 L)^{1 / 2}}
$$

where $\alpha$ is an arbitrary real number. Thus it follows that 


$$
\psi_{p}=\frac{e^{i \alpha}}{(2 L)^{1 / 2}} e^{i(x p-E t) / \hbar}
$$

Using Eq.(3.3), we get

$$
\overline{\hat{p}}=\int_{-L}^{L} \psi_{p}^{\dagger} \hat{p} \psi_{p} d x=\frac{1}{2 L} \int_{-L}^{L} p d x=p, \overline{\hat{p}^{2}}=\int_{-L}^{L} \psi_{p}^{\dagger} \hat{p}^{2} \psi_{p} d x=p^{2} .
$$

Putting Eq.(3.4) into Eq.(3.1), we deduce

$$
\Delta \hat{p}^{2}=\overline{\hat{p}^{2}}-\overline{\hat{p}}^{2}=0
$$

Using quantum uncertain relation $\Delta x \Delta p \geq \frac{\hbar}{2}$ and Eq.(3.5), we have the uncertain space

$$
\Delta x \geq \frac{\hbar}{2 \Delta p}=\infty
$$

Namely, the uncertainty property of plane wave is infinite, thus when plane wave passes the double slits, it can simultaneously pass through both Young's Double Slits and any many slits.

\section{Quantum uncertain principle's making the single particle with global property have no certain path}

Using Eq.(2.1), we have

$$
\begin{aligned}
& \varphi(\vec{p}, t)=\frac{1}{(2 \pi h)^{3 / 2}} \int_{-\infty}^{\infty} \psi(\vec{r}, t) e^{-i \vec{p} \cdot \vec{r} / \hbar} d \vec{r} \\
& =\frac{1}{(2 \pi \hbar)^{3 / 2}} \int_{-\infty}^{\infty} \frac{1}{(2 \pi \hbar)^{2}} \int_{-\infty}^{\infty} \phi\left(\vec{p}^{\prime}, E\right) e^{i\left(\vec{p} \cdot \dot{r}^{-}-t E\right) / \hbar} d \vec{p}^{\prime} d E e^{-i \vec{p}^{\prime} \cdot r^{\prime} / \hbar} d \vec{r} \\
& =\frac{1}{(2 \pi \hbar)^{1 / 2}} \int_{-\infty}^{\infty} \int_{-\infty}^{\infty} \phi\left(\vec{p}^{\prime}, E\right) \delta\left(\vec{p}-\vec{p}^{\prime}\right) e^{-i t E / \hbar} d \vec{p}^{\prime} d E \\
& =\frac{1}{(2 \pi \hbar)^{1 / 2}} \int_{-\infty}^{\infty} \phi(\vec{p}, E) e^{-i t E / \hbar} d E
\end{aligned}
$$

Namely, Eq.(4.1) is the same as Eq.(2.2), which just show their consistence. Eq.(4.1) indicates that the wave function of the coordinate representation is equivalent to the wave function of the momentum representation except for only the inverse Fourier transformation of the spatial part of a plane wave, i.e., the two kinds of wave functions of the coordinate and momentum representations are related by the inverse Fourier transformation of the spatial part of a plane wave, which shows that we may only study plane wave actions to wave functions of different representations.

On the other hand, using general uncertain relation of quantum mechanics, one has inequality (3.6). Because the plane wave function $e^{i(\vec{p} \cdot \vec{r}-t E) / \hbar}$ in Eq.(2.1) has the oscillating amplitude $\phi(\vec{p}, E)$ for some certain $\vec{p}, E$, i.e., $\vec{p}, E$ are certainty, namely $\Delta p \rightarrow 0$, according to inequality (3.6), the positions are uncertainty $\Delta x \rightarrow \infty$. Consequently, the physical logics make the particle with global property have no certain path; after superposition integration for momentum from negative infinite to positive infinite in Eq.(2.1), it still cannot give certain 
path of the particle according to the exact mathematical deduction that the stochastic superposition integration in Eq.(2.1) cannot give certain path, in reality, the particle wave does pass the Young's double slits by its subwaves, i.e., by the evolution from Eq.(2.1) to Eq.(2.3), as shown in fig. 2.

Consequently, we discover it is the uncertain principle of quantum mechanics that makes the single particle with global property have no certain path, and then makes the subwaves of the single particle simultaneously do pass the double slits.

\section{A general theory of interference pattern appearance from a kind of quantum probabilistic entanglement}

Furthermore, the two subwaves of Eq.(2.3) are entanglement, they may form interference of subwaves, see Fig. 2, their entanglement wave function is

$$
\psi(\vec{r}, t)=c_{1} \psi\left(\vec{r}_{1}, t\right)+c_{2} \psi\left(\vec{r}_{2}, t\right),\left|c_{1}\right|^{2}+\left|c_{2}\right|^{2}=1 \quad .
$$

Using Eq.(5.1), we get the probabilistic density

$$
\begin{aligned}
& \rho(\vec{r}, t)=\psi^{\dagger}(\vec{r}, t) \psi(\vec{r}, t)=\left|c_{1} \psi\left(\vec{r}_{1}, t\right)+c_{2} \psi\left(\vec{r}_{2}, t\right)\right|^{2} \\
& =\left|c_{1} \psi\left(\vec{r}_{1}, t\right)\right|^{2}+\left|c_{2} \psi\left(\vec{r}_{2}, t\right)\right|^{2}+c_{1}^{\dagger} \psi^{\dagger}\left(\vec{r}_{1}, t\right) c_{2} \psi\left(\vec{r}_{2}, t\right)+c_{2}^{\dagger} \psi^{\dagger}\left(\vec{r}_{2}, t\right) c_{1} \psi\left(\vec{r}_{1}, t\right)
\end{aligned}
$$

When one measures that the single particle passes slit $\mathrm{s}_{1}$, then $\left|\psi\left(\vec{r}_{1}, t\right)\right|^{2}=1$, thus he must get $\psi\left(\vec{r}_{2}, t\right)=0$ because of Eq.(5.1), consequently, using Eq.(5.2), we deduce

$$
\rho(\vec{r}, t)=\rho_{1}(\vec{r}, t)=\left|\psi\left(\vec{r}_{1}, t\right)\right|^{2}=1 .
$$

Namely, there is no interference term in Eq.(5.3), which means that the interference disappears.

When one measures that the single particle wave passes slit $\mathrm{s}_{2}$, the studies are similar.

When no one measures which way slit that the single particle wave passes, then Eq.(5.2) shows that there are interference terms.

Therefore, we discover a kind of entanglements with Wheeler's delayed choice (after a particle passes through the slits, extracting "which path" information at the slits can seem to retroactively alter its previous behavior, refering to [22]), i.e., quantum probabilistic entanglement, e.g., from Eq.(5.1).

Because of using the usual entanglement theory, when the wave function of the system is expressed as

$$
\psi(\vec{r}, t)=c_{12} \psi_{1}\left(\vec{r}_{1}, t\right) \psi_{2}\left(\vec{r}_{2}, t\right),
$$

the system has no entanglement. For the other cases, there are entanglements. For example, Eq.(5.1) has entanglement from quantum probabilistic conservation.

Consequently, this paper finds the quantum probabilistic entanglement from Eqs.(5.1)-(5.3).

Therefore, the crisis of the single particle's simultaneously passing through Young's Double Slits is solved.

\section{Discussions, summary and conclusions}

Global property of particle does not allow a single particle to pass through Young's double slits at the same time, in reality, the interference of individual particle wave is observed, which 
just shows the single particle wave simultaneously does pass the double slits, but all theories up to now cannot solve the crisis of the single particle's simultaneously passing the double slits e.g., see $[11,20,27]$.

The particle plane wave passes through Young's double slits and cause interference, the wave nature of the particle passes through the double slits by the uncertain principle. Its specific novel physical details are reflected out by concrete computation of this paper. Namely, this paper proves that quantum uncertain principle makes the single particle with global property have no certain path, and then makes the single particle wave simultaneously do pass the double slits.

This paper shows a general theory of interference pattern appearance from a kind of quantum probabilistic entanglement, i.e., quantum entanglement can also be applied to illustrate that the disappearance of interference patterns is the result of entanglement between diffraction subwaves of the single particle.

The two subwaves of eq.(2.3) are entanglement, they may form interference of subwaves, their entanglement wave function is eq.(5.1).

When one measures that the single particle passes slit $\mathrm{s}_{1}$, then $\left|\psi\left(\vec{r}_{1}, t\right)\right|^{2}=1$, thus he must get $\psi\left(\vec{r}_{2}, t\right)=0$ because of quantum probabilistic entanglement eq.(5.1). Consequently, using eq.(5.2), we deduce there is no interference term in eq.(5.3), which means that the interference disappears.

When no one measures which way slit that the single particle wave passes, then eq.(5.2) shows that there are interference terms.

Therefore, we find a kind of entanglements with Wheeler's delayed choice, i.e., quantum probabilistic entanglement from eqs.(5.1)-(5.3).

Quantum entanglement endows microscopic particles with nonlocal characteristics, this is the feature that classical particle concepts don't have at all, nonlocality is the result of the basic assumptions of quantum mechanics, which is naturally proved in this paper.

Consequently, the crisis of the single particle's simultaneously passing through Young's Double Slits is solved in two methods of both quantum uncertain principle and quantum probabilistic entanglement, respectively.

In the photoelectric effect, one needs to think of light as particles, otherwise the electrons will be unable to be beaten out by light wave. That is, in the wave-particle duality, the particle property is only considered, wave property is not considered, and then the photoelectric effect is able to be explained. Similarly, in Young's double slit experiment, the particle property is only considered, it is impossible for a single photon to pass through two slits at the same time. But if we just think about wave property, it is perfectly natural for the single photon wave to pass through the double slits at the same time.

Experimentally we do find that the single photon wave of light travels through the double slits at the same time. That is to say, in the reality of microphysics, for wave-particle duality of matter, just thinking about one way can explain the problem. If thinking about both, people can't explain their problems.

In photoelectric effect and Young's double slit experiment, the single photon particle can hit electrons out, and the single photon wave can go through Young's double slits at the same time, therefore, this paper novelly discovers that the two cases are complementarily equivalent (i.e., the 
ability of the single photon to hit electron out is complementarily equivalent to the ability of wave of the single photon to pass through Young's double slits at the same time in wave-particle duality).

So, in quantum physics, when thinking about wave property, people cannot think about particle property. Namely, if one considers the particle property, he cannot consider wave property. Thus the single photon wave's passing through Young's double slits at the same time is naturally explained. The photoelectric effect and the single photon wave through Young's double slits at the same time give us a deeper understanding of the wave-particle duality of the microscopic particle world. But what we want to emphasize here is that the single photon wave simultaneously goes through Young's double slits, not the single photon particle simultaneously goes through Young's double slits. As we said in the photoelectric effect, it is not a single photon wave that blows out an electron, it is the single photon particle that blows out an electron.

Similarly, quantum particles such as electrons, neutrons, protons and so on mean that wave of their single quantum particle can simultaneously pass through Young's double slits, rather than individual quantum particle may pass through Young's double slits at the same time. This indicates that this effect is a quantum effect, because quantum effect has wave-particle duality. When considering wave property, we cannot consider particle property, because the whole quantum particle system is behaving like wave property (Just as in the photoelectric effect, considering the particle nature of the system, people cannot consider wave property, otherwise the photoelectric effect cannot appear). Namely, the single quantum particle can show as either particle or wave, but cannot be observed as both at the same time for a physics process.

Classical particles mean the effects relevant to their Planck's constant, i.e., quantum effects, e.g., wave properties, are negligible. That is, the single classical particle cannot simultaneously pass through Young's double slits, while wave of the single quantum particle can pass through Young's double slits at the same time, these can be viewed as the objective criteria for distinguishing classical and quantum particles. Consequently, we discover the objective criteria for distinguishing classical and quantum particles for the first time. Thus, the objective criteria not only, for the first time, objectively give the applicable realm of quantum mechanics that quantum particles satisfy, but also are key and very useful in quantum physics and science, which will greatly help us to distinguish, investigate and understand physics world and science. Therefore, for the single particle to simultaneously pass through Young's double slit experiment, needing to divide the studies into classical and quantum cases, the single classical particle cannot pass, while the single quantum particle wave may pass, then all the problems are solved.

For example, using the discovered objective criteria for classical and quantum particles, the crisis of the example appearing in fig. 1 above does not exist. Because as a person he is a macroscopic classical object, there is no such wave property to produce the physical phenomenon in fig.1.

Furthermore, eq.(2.1) shows that the wave function of the momentum representation becomes the amplitude of the plane wave motion, that is, a plane wave with values of momentum and energy, respectively, of wave function of the 4-dimensional momentum representation moves as a plane wave with the amplitude of the wave function of the momentum representation. So when considering the motion of this complex plane wave, people needn't to consider the particle 
nature. Because the momentum representation wave function shows the global nature of the system, and the function is independent of spacetime coordinates. Furthermore, this whole property for a single particle is the property of the single particle, i.e., particle nature. Namely, we discover a new physics mechanism (from eq.(2.1)) that the particle property is transformed into the amplitude of a plane wave and the amplitude passes with the plane wave through the double slits at the same time, we call the new physics mechanism as the mechanism of passing the double slits of the plane wave with the amplitude of 4-dimensional momentum representation state vector in eq.(2.1). Actually, when doing the second quantization of eq.(2.1), the momentum representation wave function will change as particle number representation operators [24], which just show particle property and is consistent with the studies of this paper.

When considering particle properties, people cannot to use the wave functions in the spacetime coordinate system, just use the 4-dimensional momentum representation wave function that reflects its overall properties (which is just particle property for the single particle). Namely, the inverse transformation $e^{-i(\vec{p} \cdot \vec{r}-t E) / \hbar}$ of equation (2.1) is taken and integrates for 4-dimensinal spacetime coordinates to eliminate the contribution of plane waves $e^{i(\vec{p} \cdot \vec{r}-t E) / \hbar}$ in order to obtain 4-dimensional momentum representation state vector of reflecting the global particle property. And the momentum and energy have a probability density distribution of the momentum representation wave function. So the whole theoretical system of rigorous mathematical and physical descriptions of wave-particle duality is self-consistent.

Now the question is when taking the particle property and when taking the wave property. Therefore, there must be a principle, namely, the principle of self-adaptive emergence of wave-particle duality, which means when a particle is faced with the experimental equipment that it is suitable to show particle property, then particle property is shown, e.g., the photoelectric effect shows particle nature; when a particle is faced with the experimental equipment that it is suitable to show wave property, then wave property is shown, such as Young's double slit experiment displays wave interference. Therefore, this paper, for the first time, discovers the principle of self-adaptive emergence of wave-particle duality, and the single quantum particle can show as either particle or wave, but cannot be observed as both at the same time for a physics process, which are very useful in quantum physics, quantum communication, quantum calculation and so on.

According to the principle of self-adaptive emergence of wave-particle duality, the wave or particle behavior of the microscopic particles depends on the used observation system.

For example, when a beam laser splits through a flat crystal into reflected light a and refracted light $b$, the two beam lasers of $a$ and $b$ appear to be two entangled subbeam lasers. The measurements of the two beam lasers are two specific physical processes, thus, the two beams of light can behave either as waves or as particles, depending on the instruments being used to observe them. There are four cases: 1. Both $\mathrm{a}$ and $\mathrm{b}$ beam lasers are measured with two wave-measuring instruments (such as Young's double-slit device), we will get the wave property of the two sub-light waves. 2. For the two beam lasers of a and b, the wave and particle properties of the two beam lasers will be measured by respectively wave instrument and particle instrument (such as the photoelectric effect device), we will get the wave and particle properties of the two sub-beam lasers respectively. 3. For the two beam lasers of a and $b$, the particle and wave 
properties of the two beam lasers will be measured by respectively particle instrument and wave instrument, we will get the particle and wave properties of the two sub-beam lasers respectively. 4 . Both $a$ and $b$ beam lasers are measured with the measuring particle instruments, we will get the particle property of the two sub-beam lasers.

Cases 2 and 3 reflect a symmetry of the reflected and refracted lights in showing the wave-particle duality.

The theory here not only has been proved by the current experiments, e.g., Refs. [28, 29], but also gives the more direct theoretical explanations to the current experiments, e.g., Refs. [28, 29].

Let's extend these to neutron particles of Fermions.

When the monochromatic neutrons are separated into detector 1 and detector 2 subwaves after passing through the complete crystal sheet of silicon [30], as shown in fig. 3 ( about the more details of neutron interferometry refer to Ref.[30] ), i.e., fig. 4 ( is a part of fig. 10.2 in Ref.[30], but we directly extensively use fig. 4 to investigate wave-particle duality of neutrons ),

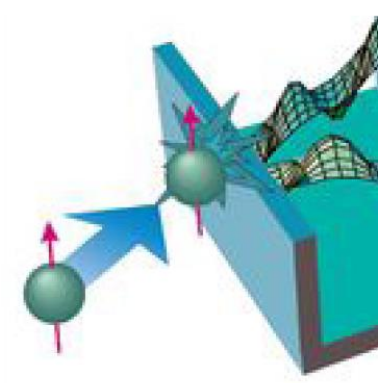

Fig.3 Monochromatic neutrons are separated into two subwaves

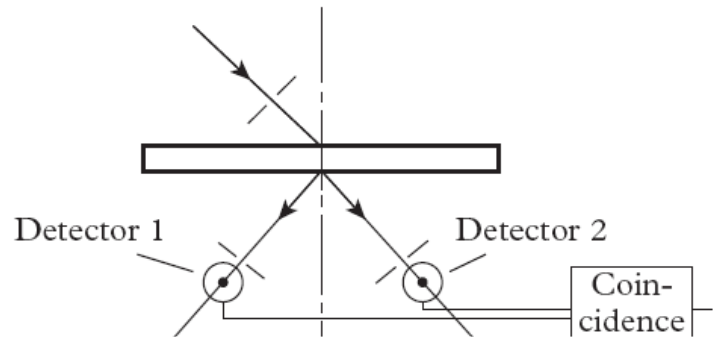

Fig. 4 Monochromatic neutrons are splited as 1,2 subwaves after passing through the complete crystal sheet of silicon

then the subwaves behind the complete crystal sheet of silicon can be seen as two entangled wavelets. The measurement of these two beams of neutrons are two specific physical processes, thus, these two subwaves can behave as waves and/or particles, depending on the instruments used to observe them. There are four cases: 1 . In both regions of detector 1 and detector 2 we measure them with instruments that measure wave property, we will get wave property of the two subwaves. 2. For the two beam neutrons of detector 1 and detector 2, the wave and particle properties of the two beams will be measured by respectively wave instrument and particle instrument, we will get the wave and particle properties of the two sub-beams respectively. 3. For the two beam neutrons of detector 1 and detector 2, the particle and wave properties of the two beams will be measured by respectively particle instrument and wave instrument, we will get the particle and wave properties of the two sub-beams respectively. 4. Both detector 1 and detector 2 beams are measured with the measuring particle instruments, we will get the particle property of the two sub-beams.

The coincidence in fig. 4 means that there is the coincidence count rate fluctuation in both detectors [30] and that we need to adjust them as similar or identical state as possible so that we can equivalently do the symmetry experiments.

Cases 2 and 3 reflect a left-right symmetry about the experiments of the wave-particle duality of the microscopic neutron particles.

Therefore, these experiments' predictions of the wave-particle duality of the system of one neutron beam splitting into two sub-beam systems can be confirmed by future experiments. 
So the microscopic wave-particle duality particles that we're investigating can be either bosons or fermions. Therefore, we have generalized them to the general case.

For other general cases of wave-particle duality, people can similarly study, thus, we won't repeat here.

Consequently, using the principle of self-adaptive emergence of wave-particle duality, this paper gives both direct explanations to the current experiments and new predictions of new some experiments for wave-particle duality.

All these studies are completely consistent with all the current physical experiments. Thus, the hard crisis of the single particle's passing through Young's double slits at the same time, which have been plaguing physicists in the whole world up to now for decades, is solved. Consequently, all current articles, (text)books and concepts of physicists relevant to the results of this paper would be updated and supplied.

Acknowledgments: The work is supported by the U.S. Department of Energy, contract no. DE-AC02-05CH11231, NSF through grants PHY-08059, DOE through grant DEFG0291ER40681 and National Natural Science Foundation of China (No. 11875081 ).

\section{References}

[1] French, A.P., Taylor, Edwin F. An Introduction to Quantum Physics. Norton, (1978), ISBN 978-0-393-09106-9.

[2] Feynman, Richard P., Robert, B. Leighton, Matthew Sands. The Feynman Lectures on Physics. Vol. 3. Addison-Wesley, (1965), ISBN 978-0201021189.

[3] Lederman, Leon M., Christopher, T. Hill. Quantum Physics for Poets. US: Prometheus Books. pp. 102-111. (2011), ISBN 978-1616142810.

[4] Feynman, Richard P. The Feynman Lectures on Physics. 3:Quantum Mechanics p.1-1, (1965), "There is one lucky break, however - electrons behave just like light.".

[5] Davisson, C. J. The diffraction of electrons by a crystal of nickel. Bell System Technical Journal. 7 (1928) 90-105. doi:10.1002/j.1538-7305.1928.tb00342.x.

[6] Feynman, Richard P. The Feynman Lectures on Physics. 3:Quantum Mechanics, (1965).

[7] Merli, P G,, Missiroli, G F., Pozzi, G. On the statistical aspect of electron interference phenomena. American Journal of Physics. 44 (1976) 306-307.

[8] Rosa, R. The Merli-Missiroli-Pozzi Two-Slit Electron-Interference Experiment. Physics in Perspective. 14 (2012) 178-194.

[9] The most beautiful experiment, Physics World 2002.

[10] Frabboni, S., Gabrielli, A. et al. "The Young-Feynman two-slits experiment with single electrons: Build-up of the interference pattern and arrival-time distribution using a fast-readout pixel detector". Ultramicroscopy. 116 (2012) 73-76. doi:10.1016/j.ultramic. 2012.03.017. ISSN 0304-3991.

[11] Science Advances. 03 May 2019: Vol. 5, no. 5, eaav7610 DOI: 10.1126/sciadv.aav7610

[12] Greene, B. The Fabric of the Cosmos: Space, Time, and the Texture of Reality. Random House LLC. p. 90 (2007). ISBN 978-0-307-42853-0.

[13] Donati, O, Missiroli, G. F., Pozzi, G. "An Experiment on Electron Interference". American Journal of Physics. 41 (1973) 639-644.

[14] INairz, O., Brezger, B., Arndt, M., Zeilinger, A. Diffraction of Complex Molecules by Structures Made of Light. Phys. Rev. Lett. 87 (2001) 160401.

[15] Boscá, Díaz-Pintado, María, C. (29-31 March 2007). Updating the wave-particle duality. 
15th UK and European Meeting on the Foundations of Physics. Leeds, UK. Retrieved 21 June 2008.

[16] Bartell, L. Complementarity in the double-slit experiment: On simple realizable systems for observing intermediate particle-wave behavior. Physical Review D. 21 (1980) 1698-1699.

[17] Zeilinger A. Experiment and the foundations of quantum physics. Reviews of Modern Physics. 71 (1999) S288-S297.

[18] Mittelstaedt, P., Prieur, A., Schieder, R. Unsharp particle-wave duality in a photon split-beam experiment. Foundations of Physics. 17 (1987) 891-903.

[19] Greenberger, D.M., and Yasin, A. Simultaneous wave and particle knowledge in a neutron interferometer. Physics Letters. A 128 (1988) 391-4.

[20] Arndt, M., Nairz, O., Julian, V.-A,, Keller, C., et al. Wave-particle duality of C60 molecules. Nature. 401(1999) (6754): 680-682. doi:10.1038/44348.

[21] Schouten, H.F., Kuzmin, N., Dubois, G., Visser, T.D., Gbur, G., Alkemade, P. F.A., Blok, H., Hooft, G.W., Lenstra, D., Eliel, E.R. Plasmon-Assisted Two-Slit Transmission: Young's Experiment Revisited. Phys. Rev. Lett. 94 (2005) 053901.

[22] Jacques, V., et al. "Experimental realization of Wheeler's gedanken experiment." Science $\mathbf{3 1 5}$ 966 (2007).

[23] Hey Tony. The New Quantum Universe. Cambridge University Press (2003).

[24] Sakurai, J. J., Napolitano, J. Modern quantum mechanics. 2nd Edition. Addison-Wesley (2011).

[25] https://www.thoughtco.com/huygens-principle-2699047.

[26] Huang, C. and Huang, Yong-Chang. Unification theory of classical statistical uncertainty relation and quantum uncertainty relation and its applications. Physics Letters. A 375 (2011) 271.

[27] Holstein, Barry R. Topics in Advanced Quantum Mechanics. Dover Publications (2014).

[28] J. S. Tang, Y. L. Li, X. Y. Xu, G. Y. Xiang, C. F. Li, G.C. Guo, Realization of quantum Wheeler's delayed-choice experiment, Nature Photonics, 6 (2012) 600.

[29] Kai Wang, Qian Xu, Shining Zhu \& Xiao-song Ma, Quantum wave-particle superposition in a delayed-choice experiment, Nature Photonics, 13 (2019) 872.

[30] Raoch H., Werner S. A., Neutron interferometry, 2nd Ed., Oxford University Press, 2015. 